\begin{tabular}{|c|c|}
\hline Title: & $\begin{array}{l}\text { A Custom, High-Performance Real Time Measurement and Control System for } \\
\text { Arbitrary Power Electronic Systems in Academic Research and Education }\end{array}$ \\
\hline Authors: & Christian Axtmann, Miriam Boxriker, Michael Braun \\
\hline Institute: & $\begin{array}{l}\text { Karlsruhe Institute of Technology (KIT) } \\
\text { Elektrotechnisches Institut (ETI) } \\
\text { Electrical Drives and Power Electronics }\end{array}$ \\
\hline Type: & Conference Proceedings \\
\hline Published at: & $\begin{array}{l}2016 \text { 18th European Conference on Power Electronics and Applications (EPE'16 ECCE } \\
\text { Europe), Karlsruhe, } 2016 \\
\text { Publisher: IEEE, Piscataway (NJ) } \\
\text { Year: } 2016 \\
\text { ISBN: } 978-9-0758-1524-5 \\
\text { Pages: } 1-11\end{array}$ \\
\hline Hyperlinks: & DOI: $\underline{10.1109 / E P E .2016 .7695482}$ \\
\hline
\end{tabular}

(c) 2016 IEEE. Personal use of this material is permitted. Permission from IEEE must be obtained for all other uses, in any current or future media, including reprinting/republishing this material for advertising or promotional purposes, creating new collective works, for resale or redistribution to servers or lists, or reuse of any copyrighted component of this work in other works. 


\title{
A Custom, High-Performance Real Time Measurement and Control System for Arbitrary Power Electronic Systems in Academic Research and Education
}

\author{
Christian Axtmann, Miriam Boxriker, Michael Braun \\ Karlsruhe Institute of Technology \\ Kaiserstrasse 12 \\ Karlsruhe, Germany \\ Phone: +49 (0) 721-608 41782 \\ Fax: +49 (0) 721-358854 \\ Email: christian.axtmann@kit.edu \\ URL: http://www.eti.kit.edu
}

\section{Keywords}

$\ll$ Education Tool $\gg, \ll$ Signal Processing $\gg, \ll$ Digital Control $\gg, \ll D S P \gg$

\begin{abstract}
In high-quality education, topics concerning the behavior and control of electrical machines and power electronics have to be taught not only theoretically but also in a practical manner. Hard- and software tools are necessary to fulfil this obligation. Nevertheless, commercial systems partially lack of functionality or full accessibility to implement custom solutions, which is obligatory in the research and education domain. Therefore, a digital signal processing system is presented, that allows full modification of every used device, both in hardware and in software.
\end{abstract}

\section{Introduction}

The Institute of Electrical Engineering (ETI) is responsible for the academic education in electrical drives and power electronics at the Karlsruhe Institute of Technology (KIT). To offer high-quality research and education, it is essential to teach students in all important domains, such as dimensioning of power electronics and electrical machines, controlling them separately or in a system and measuring machines and power electronics on a test bench, Fig. 1. Therefore, it is evident that the demands, which have to be fulfilled by hard- and software tools not only match this diversity, but also have to be an integral part yet. Furthermore, there are special requirements, which emerge in educational use: Firstly, the needed system has to be cost-efficient to be able to provide it to many students at the same time. Secondly, it needs to be easy accessible in order to quickly change control mechanisms or to establish new systems. Last but not least, the whole system needs to be easily reproducible when new systems are needed.

Although there are providers of commercial measurement software and hardware for the different applications (e.g. as used in [1]), they do not satisfy all of the specifications particularly asked in an educational environment. One of the most striking disadvantages is the encapsulation of the software and hardware to a point where it is not accessible anymore. This leads to a reduced adaptability in new research fields or new tasks in education, like for example modular multilevel converters [2] or power hardware-in-the-loop systems [3]. Sometimes, this can be avoided by purchasing add-ons to hard- or software. However, this impacts the cost-aspect, which disqualifies these commercial solutions. Summing up, there is no possibility to fulfill all requirements under respect to the costs of the system.

A remedy is presented in this paper: a custom digital signal processing system for universitary use (DSPSystem). Since it is developed entirely in house, every part is completely accessible for individual change 


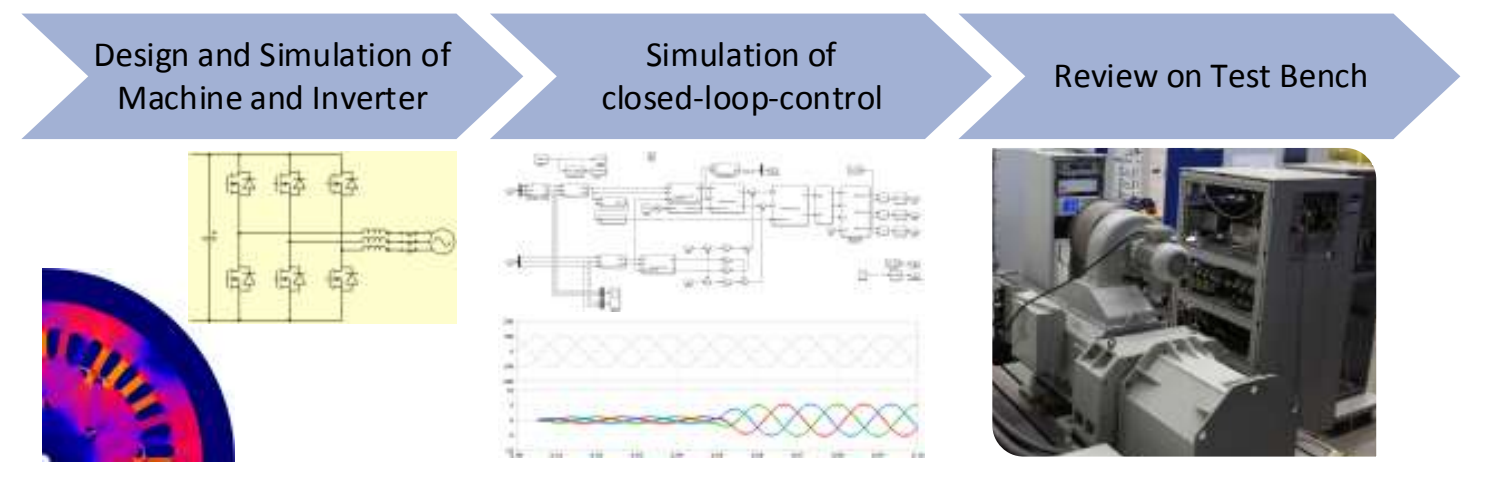

Fig. 1: The educational contents in electrical engineering match the whole design cycle of a product. Using the example of an electrical traction system, these are design, simulation and real examination of both machine and power electronics. The proposed DSP-System and Code Generation support the step from simulation to real world.

by moderate costs. The whole system is real-time capable, programmable, modular, adaptive and can be coupled with different, well-known software. That means that the DSP-System is a multifunctional tool. An additional benefit is that the students are also able to gain insight into signal processing by further developing the DSP-System during workshops and scientific theses respectively. Considering that signal processing has become an integral part of power electronics, this is another great advantage. In the following chapters the mentioned DSP-System by itself and its use in academic work will be introduced. A brief overview of the technical key features is given, followed by an explanation how the system can interact with Matlab/Simulink or LabView. Further, its current use in academic education and research is described.

\section{Digital Signal Processing System}

The proposed digital signal processing system (DSP-System) is mainly used for closed-loop control systems of power converters, but can also be used for any measurement or sequential control tasks. An exemplary control scheme that can be arranged with the system is shown in Fig. 2. It depicts a common electric drive system with closed-loop control. By means of this figure, the hard- and software of the proposed DSP-System is explained.

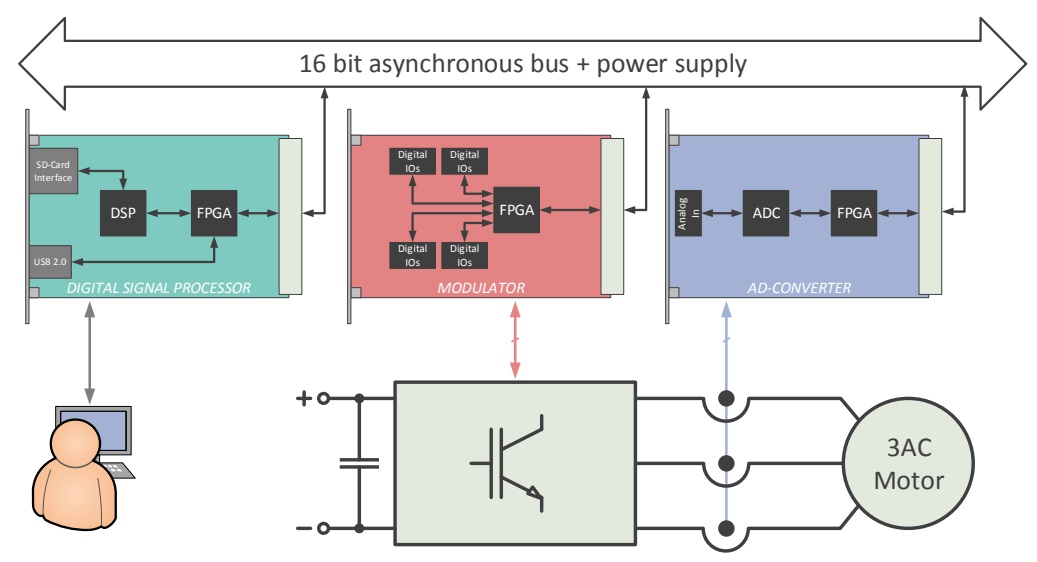

Fig. 2: An exemplary control scheme for an electric drive system. The digital signal processor runs the control algorithm, whereas the modulator and the analog-to-digital-converter interface with the power converter. 

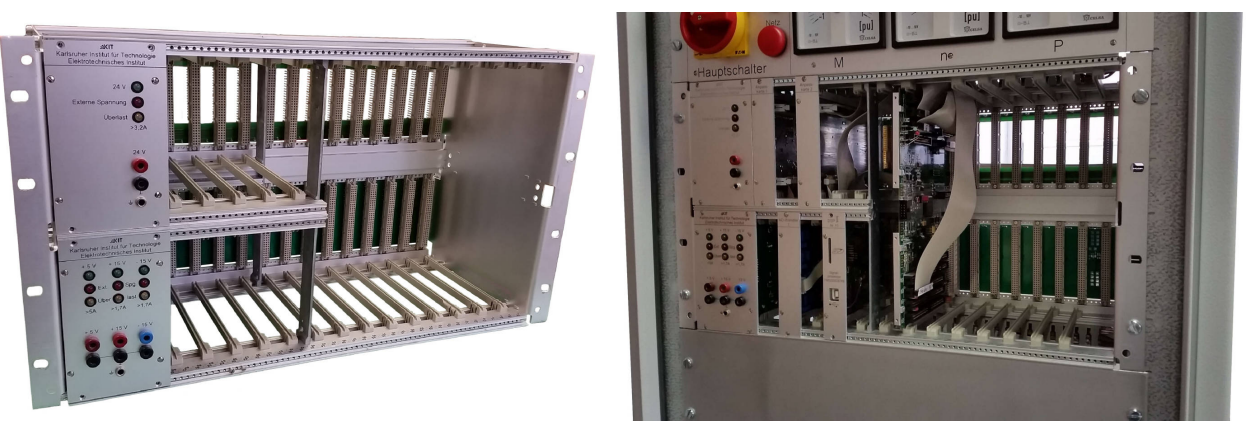

Fig. 3: Left: An empty subrack (19" / 6U) of the DSP-System. The backplane and the two power-supply units $(5 \mathrm{~V}, 15 \mathrm{~V}, 24 \mathrm{~V})$ can be seen. Right: A partially populated subrack, already built into a control cabinet.

To achieve maximum modularity, all system components consist of single or double euroboards $(160 \mathrm{x}$ $100 \mathrm{~mm}$ resp. $160 \times 223 \mathrm{~mm}$ ) that provide the various functionalities as subassemblies. All cards have a 64-pin connector according to DIN 41612 at the rear edge through which they are connected to the power supply as well as an asynchronous communication bus. This bus is 16 bit wide and can address up to 1024 participants. It features full flow control as well as two system-wide interrupts. The maximum data rate is about $50 \mathrm{Mbit} / \mathrm{s}$, which is satisfying hard real-time requirements. Because of the standardized dimensions of the cards, subracks can be used as seen in Fig. 3. One 19-inch subrack houses up to 28 subassemblies, two power supply units and a backplane to distribute both power and bus signals. Only the 14 slots on the bottom row have access to the bus, the upper 14 slots only connect to the power supply and can be used for a customized pinout.

The core of the whole DSP-System is the main computing board that holds the digital signal processor itself. Currently, there are two board versions in use with different signal processors made by Texas Instruments. Their product names and key features are given in Table I. The cards are depicted in Fig. $4 a$ ) and b). Field programmable gate arrays (FPGAs) on the processor boards manage data exchange via the asynchronous bus or via a USB connection on the front side to a host computer. From there the DSPs can be freely programmed. To complete a closed-loop control, further components are needed, to acquire and emit data.

Table I: Comparison of the key features of the two digital signal processor boards

\begin{tabular}{ccc}
\hline & TMS320VC33 & TMS320C6748 \\
\hline Flash-Memory & $512 \mathrm{kB}$ & $8 \mathrm{MB}$ (+ SD-Card) \\
RAM & $1 \mathrm{MB}$ & $34 \mathrm{MB}$ \\
USB-Connection & $8 \mathrm{Mbit} / \mathrm{s}$ (USB 1.1) & $40 \mathrm{MBit} / \mathrm{s}$ (USB 2.0) \\
Cache & $136 \mathrm{kB}$ & $200 \mathrm{kB}$ \\
Clock Rate & $150 \mathrm{MHz}$ & $456 \mathrm{MHz}$ \\
Processing Power & $150 \mathrm{MFLOPS}$ & $2746 \mathrm{MFLOPS}$ \\
\hline
\end{tabular}

Usually, it does not matter if electric or non-electric values are measured, since there are sensors that convert their measured values to voltage signals. Thus, a subassembly that can measure and quantify these voltages is needed. Therefore, analog-to-digital-converter cards have been developed (Fig. 4c) and steadily improved. At their current development status, they feature a 4-channel Analog-to-DigitalConverter IC (ADC) with up to $1.5 \mathrm{MS} / \mathrm{s}$ per channel and an FPGA. This FPGA not only interfaces the $\mathrm{ADC}$ to the main computing board via the asynchronous bus, but also provides several conveniences to the user, such as automatic averaging over one modulation period or multiplexing of up to 16 channels for slowly changing signals. In addition, further acquisition cards have been developed, e.g. for resolver- or incremental encoder-evaluation, which are also based on FPGAs and interface directly to the asynchronous bus. 

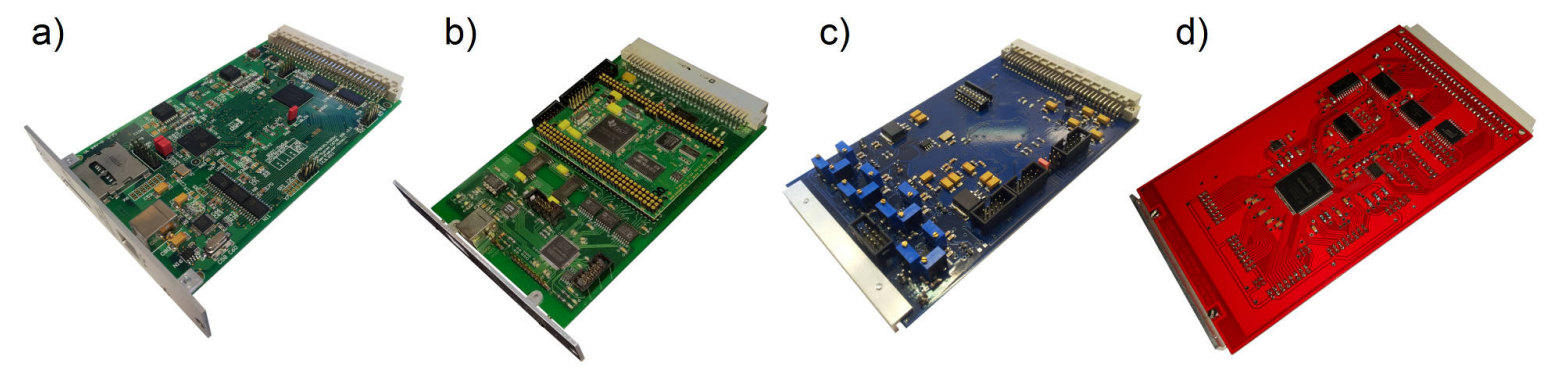

Fig. 4: a) and b) The two main computing boards, equipped with digital signal processors by Texas Instruments (green), c) an analog-to-digital converter board with 4 channels @ 1.5MS/s each (blue), d) a card holding a FPGA as a modulator (red). All cards are freely programmable and were developed in various bachelor and master theses.

Finally, a modulator is needed that converts the actuating variable of the feedback control algorithm to output signals, e.g. timed gate signals for a three-phase bridge circuit. For this purpose several custom FPGA boards were developed, called modulator boards, seen in Fig. 4d). The boards only hold an FPGA by Altera that is also linked to the asynchronous system bus. Its remaining IOs are directly wired to several plug-in connectors. The main task of this FPGA is determining the switching times by sine-triangle pulse-width modulation (SPWM) for the semiconductor-switches. Besides, it can generate signals for e.g. main and auxiliary contactors or receive signals from e.g. push-buttons. Because of its simplicity in hardware and the free programmability, this board not only serves to generate switching signals, but also provides the possibility to synthesize common interfaces, like RS-232 or SPI.

The most sophisticated FPGA card is the so called high performance modulator card (HMK). Compared to the "common" modulator card with about 6000 logic elements and 55 user-configurable IOs, the HMK features nearly 40000 cells and 275 IOs while still maintaining a regular interface to the introduced asynchronous bus. The key benefit of this device is the availability of its own, dedicated peripheral subassemblies, such as a 8-channel analog-to-digital converter or a 4MiB RAM-Module, among others. These connect directly to the IOs, discarding the need for a bus system. By this means, one has the ability to relocate control algorithms in the FPGA. In cascaded control structures, these are the inner control loops (e.g. current control loops) preferably. This shortens the data path between measurement, control algorithm and gate-signal generation, allowing much shorter control periods. It could be proved, that even nonlinear, lookup-table-based control algorithms can be executed with a control frequency of $1.5 \mathrm{MHz}[4]$.

\section{Monitoring Control Tool and MATLAB Code Generation}

Another important part of the whole system is its connection to a host computer and the software running on it. On the one hand the host computer needs a host program that allows for booting the signal processor, entering desired values and visualizing measured data. On the other hand the host computer is needed to write and compile the program source code for the signal processor. The first task is accomplished by custom computer software, called the Monitoring Control Tool. This software has been written in LabView by a student in his master thesis. It follows NIs Instrument Driver Guidelines [5] that enables the DSP-System to interface with common LabView software and enables the user to interact with the DSP-System.

As it can be seen in Fig. 5 on the left, the user frontend provides several ways to visualize measured data as waveforms or as simple digits. As one benefit of the in-house implementation, it is possible to customize the surface of the program to better fit the users needs. Furthermore, a lot of high quality test equipment features direct interface to LabView, so that these instruments can be evaluated and logged simultaneously. With such a setup, automatic measurements can be carried out, for example measuring the efficiency of a machine or power converter in different operating points. Providing a development environment for the DSP-System is the second task of the host computer. This can be accomplished by 

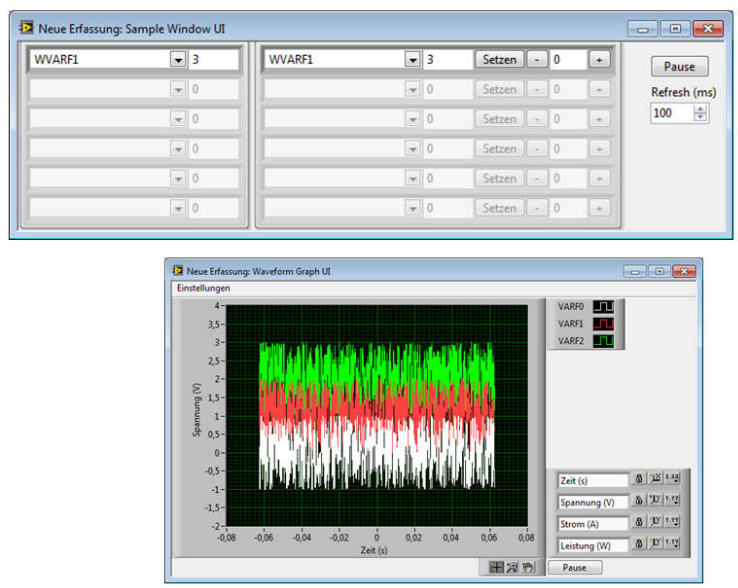
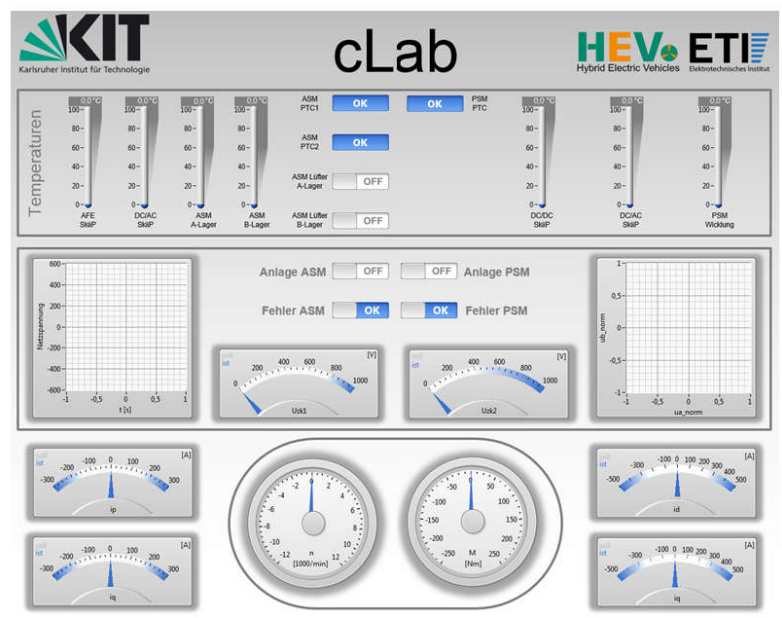

Fig. 5: Screenshots of the Monitoring Control Tool. Measured or setpoint values can be visualized in form of digits or waveforms (left), but the user front end can also be individualized to fit special needs (right).

two methods. A C-compiler is provided by Texas Instruments for the signal processor, providing the possibility to program the whole control task in native C. However, a more sophisticated way to program the signal processor is using Matlab Simulink and its included C-Coder [6]. Together with a customized blockset and Simulink-extension, it is possible to convert a control algorithm of an already working simulation to executable code for the DSP-System. The mechanism was developed in [7].

This leads to another appealing feature: it enables rapid control prototyping by transferring the projected control software models directly from simulation to the test bench. The emerging advantages like the fast validation and the flexibility of control strategies are described in [8]. This process is also available for FPGAs as Matlab Simulink also features a VHDL Coder.

In contrast to $\mathrm{C}$ or VHDL, graphical programming languages, such as Simulink, support the user because of its easy understandable presentation of signal flow. But high-level languages are needed, when the algorithms have to be executed on a DSP or FPGA. Consequently, the code generation assists on two levels: On the one hand, the simulation and the development of models and algorithms can be done graphically, so it is easier to debug, to change or to adapt. On the other hand, there is no need to rewrite the software for the test bench and the DSP-System. This minimizes the error rate and the needed time to test the model.

\section{Educational Use}

Within the educational strategy of ETI, the DSP-System plays an important role by supporting it in three major aspects:

- Consolidating the students knowledge acquired in lectures and gaining first practical experience by participating in workshops and laboratory courses, which would not be possible without the DSP-System

- Further development of the DSP-System and getting insight in signal processing and programming, as a basis for controlling power electronics and electrical machines

- Starting scientific work with bachelor and master theses supported by the DSP-System in the field of power electronics and electrical machines

This also builds a continuous closed loop in developing tasks: New demands arise by applying the DSPSystem in Bachelor and Master theses which promotes constant further development of the DSP-System and the associated cards and programs. In turn, this gives the opportunity to exploiting new fields of research. Therefore, the DSP-System is fundamental to education by combining research and teaching. 

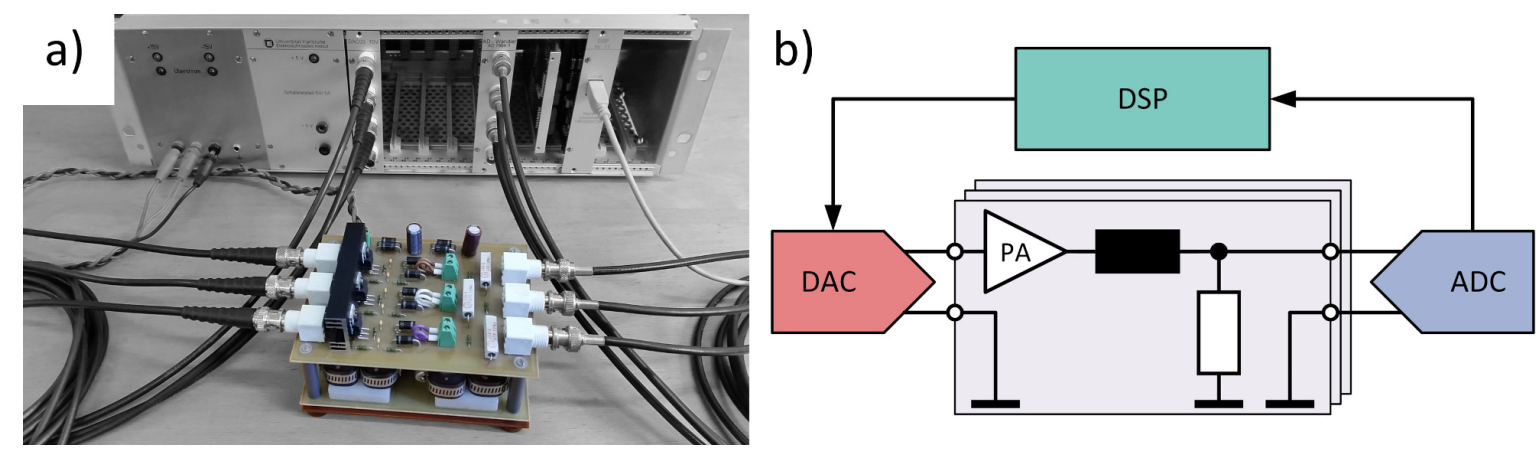

Fig. 6: a) Picture of the three-phase RL-load (inductors on lower PCB). b) simplified schematic of the RL-load and the surrounding signal processing system. The voltage of the digital to analog-converter (DAC) is fed into a power amplifier (PA). The voltage across the load resistor is measured by the analogto-digital converter. As it is proportional to the flowing current, it is an adequate test point for closed-loop current control.

The first pillar of education can be clearly seen in the developing process of the DSP-System: The first efforts to a custom signal processing solution were taken in 1994. Until then, transputers were used for real-time control systems. Since then, more than 30 diploma, bachelor and master theses were written to develop and improve the whole system. Nevertheless, these were not the only one pushing the DSP-System. Many student assistants developed parts of the system, always supervised by scientific assistants. Thereby, students did not only gain knowledge in the domain of power electronics, but also in signal processing, programming or analog and digital circuit design.

Besides research, the system itself is used in several laboratory courses for students. In these hands-on trainings, students learn to implement those control tasks that they will most likely face in later work. The offered lab courses are mostly focused on master students, who already decided to specialize in electric drives and power electronics, so the experiments are explicitly tailored to this specific field. In several preceding compulsory courses, the students already learned the needed theoretical basics. These include the behavior and control of power electronics and electrical machines, which shall be deepened in the laboratory courses by adding a practical component.

One common experiment is a current control in a rotating reference frame, which is described exemplary below. For that purpose a three-phase RL-load with integrated power amplifier was designed, see Fig. 6. In a first step, the students are supposed to get to know the DSP-System itself in a written experiment instruction. In several consecutive assignments, the students stepwise put the whole system into operation by expanding a given program structure. First, arbitrary voltages (preferably sinusoidal) are generated with the digital-to-analog converter (DAC). These can be checked with a conventional oscilloscope. In a second step, the output of the DAC is fed into the RL-load and the system response to various stimuli can be measured, first with an oscilloscope, then with the already introduced analog-to-digital converter (ADC). At this point, open-loop control of the load is possible. In the last step, the students have to design a conventional PI current controller and extend the program structure to a closed-loop control. It should be mentioned that there is no decoupling needed inside the control algorithm as the RL-load consists of three passive independent phases. However, this includes that there is no information about an angle given, so that it has to be synthesized inside the program. The ability to directly programming the DSP using floating-point arithmetic enormously simplifies all the programming.

That is only one example of the operational capability of the DSP-System and its use in education. There are many more laboratory experiments deepening the students understanding and, of course, numerous theses working or having worked with the DSP-System. 


\section{Conclusion}

In this paper a digital processing system is presented that is fully modular and freely programmable. The fact that it is completely developed in-house brings various advantages. First, nearly every given task can be fulfilled because of the modularity, scalability and free programmability. Because of the mentioned modularity, standard components are available, that can be easily fabricated and stored. Nevertheless, these standard components can be used as a base for every further development. Together with the fact that the system is relatively cheap compared to commercial pendants, but still interfaces with well-known and tested software, it depicts an outstanding tool for universitary education and research. On the one hand the system and its computer tools are used to teach the whole domain of electrical drivetrain development, on the other hand students can gain insight in the domain of signal processing, programming and circuit design, by improving the system and its components, e.g. as master thesis or student assistant work. Hence every subsequent user instantly gains benefit of these improvements.

\section{References}

[1] P. Saey et al.: Using an industrial hardware target for Matlab generated real-time code to control a torsional drive system, in 15th Conf. on Power Electronics and Applications (EPE13), Lille, France, 2013.

[2] J. Kolb: Optimale Betriebsführung des Modularen Multilevel-Umrichters als Antriebsumrichter für Drehstrommaschinen, Ph.D. dissertation, Institute of Electrical Engineering, Karlsruhe Institute of Technology, Germany, 2013.

[3] A. Schmitt et al.: A High Current, High Frequency Modular Multiphase Multilevel Converter for Power Hardware-in-the-Loop Emulation, in International Exhibition and Conference for Power Electronics, Intelligent Motion, Renewable Energy and Energy Management. PCIM Europe 2014, 2014.

[4] A. Schmitt et al.: FPGA-Based Real-Time Simulation of Nonlinear Permanent Magnet Synchronous Machines for Power Hardware-in-the-Loop Emulation Systems, in 40th Annual Conference of the IEEE Industrial Electronics Society (IECON 2014), Dallas, USA, 2014.

[5] National Instruments: (2015, November 25), Instrument Driver Guidelines [Online]. Available: http://www.ni.com/devzone/idnet/library/instrument_driver_guidelines.htm

[6] The MathWorks: (2015, November 25), Generating C Code from Your MATLAB Algorithms [Online]. Available: http://blogs.mathworks.com/loren/2011/11/14/generating-c-code-from-your-matlab-algorithms/

[7] T. Gemaßmer: Effiziente und dynamische Drehmomenteinprägung in hoch ausgenutzten Synchronmaschinen mit eingebetteten Magneten, Ph.D. dissertation, Institute of Electrical Engineering, Karlsruhe Institute of Technology, Germany, 2015.

[8] D. Abel and A. Bollig: Rapid Control Prototyping, Berlin, Germany: Springer-Verlag, 2006. 